J. Clin. Chem. Clin. Biochem.

Vol. 19,1981 , pp. $479-484$

\title{
Untersuchungen zur circadianen Rhythmik der Ausscheidung freier Katecholamine bei gestörter Nebennierenfunktion ${ }^{1}$ )
}

\author{
Von D. Ratge, E. Knoll, U. Diener und $H$. Wisser \\ Abteilung für Klinische Chemie, Robert-Bosch-Krankenhaus, Stuttgart
}

(Eingegangen am 2. Oktober 1980/23. Januar 1981)

Zusammenfassung: Die Tageszeitabhängigkeit der Urinausscheidung der Katecholamine bei Überfunktion des sympathoadrenalen Systems (3 Phäochromocytome, 1 Neuroblastom), gestörter Nebennierenrindenfunktion (M. Addison und partielle Nebennierenrindeninsuffizienz), einer Patientin mit bilateraler Adrenalektomie und 8 gesunden Probanden unter Dexamethason und einer Kontrollgruppe wurde bestimmt. Dabei wurde festgestellt, daß bei allen untersuchten Gruppen die circadiane Rhythmik der Katecholamine vorhanden war. Die Ausscheidung der Katecholamine und der Vanillinmandelsäure erscheint bei den Patienten mit einem Phäochromocytom gegenüber der Kontrollgruppe phasenverschoben. Bei den Patienten mit Uberfunktion des sympathoadrenalen Systems wurden die Amplituden der Katecholamine und der Vanillinmandelsäure sich ähnlicher, während bei gesunden Probanden die Vanillinmandelsäure und das Dopamin gegenüber Adrenalin und Noradrenalin deutlich kleinere Schwingungsbreiten zeigten.

\section{Circadian rhythm of free catecholamine excretion in disturbed adrenal function}

Summary: The daily time course of urinary catecholamine excretion was determined for hyperactivity of the sympathoadrenal system ( 3 phaeochromocytomas, 1 neuroblastoma), disturbed adrenal function (M. Addison and partial adrenal insufficiency), bilateral adrenalectomy (one patient), in 8 healthy patients receiving dexamethasone, and in a control group. All groups showed a circadian rhythm of catecholamine excretion. In the patients with phaeochromocytoma, the excretion of catecholamines and vanilmandelic acid showed a shift of phase compared with the control group. In patients with hyperfunction of the sympathoadrenal system, catecholamines and vanilmandelic acid fluctuated with similar amplitudes, whereas in healthy patients vanilmandelic acid and dopamine showed much smaller fluctuations of concentration than adrenaline and noradrenaline.

\section{Einleitung}

Es ist schon lange bekannt, daß die Ausscheidung der Katecholamine im Urin tageszeitlichen Änderungen unterliegt $(1,2)$. Selbst unter härtesten Streßbedingungen bleibt dieser Rhythmus erhalten (3). Auffallig ist, daß die Ausscheidung von Adrenälin und Noradrenalin sehr große Amplituden, die von Dopamin, Vanillinmandelșäure und Homovanillinsäurure sehr kleine Amplituden hat. Als Erklärungsmöglichkeit bietet sich der unterschiedliche Einfluß verschiedener Kompartimente an, die zur Urinausscheidung der genanniten Verbindungen beitragen. So soll das im Urin ausgeschiedene Adrenalin weitgehend aus dem Nebennierenmark, das Noradrenalin aus den sympathischen Nervenendigungen, die Vanillinmandelsäure aus mindestens 5 Kompartimenten (4) stammen. Untersucht man Patienten mit einem großen Kompartiment, z.B. einem Tumor, dann sollte man erwarten, - wenn diese Hypothese stimmt -, daß die Amplituden der Katecholamine und ihrer Metabolite einander ähnlicher werden. Eine andere Möglich- keit, den Einfluß verschiedener Kompartimente zu ermitteln, bietet die Untersuchung von adrenalektomierten Patienten. Nach Adrenalektomie sinkt nämlich die Adrenalinausscheidung im Urin auf zunächst nicht meßbare Werte (5), um dann wieder Normwerte zu erreichen. Daher wurde die Katecholaminausscheidung bei einer adrenalektomierten Patientin bestimmt, um festzustellen, ob dieses, aus einem anderen Kompartiment stammende Adrenalin, eine veränderte Zeitstruktur der Ausscheidung zeigt.

Schließlich sollte geprüft werden, ob eine veränderte Glucocorticoidsekretion zu einer Beeinflussung der Ausscheidung des Adrenalins führt. Nach Untersuchungen von Wurtman \& Axelrod $(6,7)$ läßt sich bei hypophysektomierten Ratten nach Gabe von Corticotropin oder Dexamethason die erniedrigte Aktivität der Phenylethanolamin-N-methyltransferase (EC 2.1.1) wieder

\footnotetext{
1) Diese Arbeit wurde unterstützt aus Mitteln der Robert Bosch Stiftung, Stuttgart.
} 
normalisieren. Es konnte gezeigt werden, daß Corticotropin nur über die Stimulierung der endogenen Glucocorticoidsekretion wirkt. Die Glucocorticoide sind keine Aktivatoren der Phenylethanolamin-N-methyltransferase, sondern sie erhöhen die Aktivität über eine vermehrte Enzymproteinsynthese. Aufgrund dieser Beziehung wurde bei einer Patientin mit einer partiellen Nebenniereninsuffizienz, einer Patientin mit Morbus Addison und 8 gesunden Probanden unter Dexamethason-Gabe die Tageszeitabhängigkeit der Urinausscheidung der Katecholamine bestimmt. Die Urinproben der 8 Versuchspersonen stammen aus einer anderen Studie über die Beeinflussung des Testosteron- und Lutropin-Rhythmus durch Dexamethason-Suppression (8). Als Vergleichsgruppe wurden 4 gesunde freiwillige Versuchspersonen unter Klinikbedingungen untersucht.

\section{Versuchsbeschreibung}

Vier gesunde männliche Probanden im Alter zwischen 24 und 29 Jahren wurden am Morgen des ersten Versuchstages in die Klinik einbestellt. Die Probanden muß ten während der folgenden beiden Versuchstage im Bett liegen. Aufstehen war nur zur Urinsammlung erlaubt. 2-Stunden-Urinproben wurden über 48 Stunden, beginnend mit der ersten Sammelperiode von 8.00 bis $10.00 \mathrm{Uhr}$, gesammelt. Da über einen Venenkatheter im 2stündigen Abstand, allerdings um 1 Stunde zeitverschoben, gleichzeitig Blutproben entnommen wurden, konnten die Probanden aufgrund der allstündlichen Störung die Nacht nur im Halbschlaf verbringen. Die Mahlzeiten wurden um 7.30 Uhr, 11.30 Uhr und 17.00 Uhr eingenommen. Mineralwasser stand stets zur Verfügung. Verboten waren Medikamente, Rauchen, Alkohol, Kaffee, Tee, Bananen und vanillehaltige Speisen.

Die klinischen Daten der Patienten mit einem Phäochromocytom bzw. Neuroblastom sind in Tabelle 1 wiedergegeben. Die Patienten mit dem Phäochromocy tom wurden präoperativ untersucht. Bei dem Patienten mit dem Neuroblastom handelt es sich um ein Rezidiv nach operativer Entfernung des Primärtumors.

Die Patientin G. M. verweigerte eine Operation. Sie wurde 9 Jahre konservativ behandelt. Während der Urinsammelperiode lag sie auf der Intensivstation und erhielt pro Tag folgende Medikamente: Diazepam ( $2 \times 5 \mathrm{mg})$, Digoxin $(0,2 \mathrm{mg}$ i.v.), Verapamil (5 mg i.v.), Triflupromazin (10 mg i.v.) und Metoprolol $(4 \times 50 \mathrm{mg})$, so daß dic gemessenen Werte mit Vorsicht interpretiert werden müssen.

Die Patientin D. A. war wegen eines Morbus Cushing 2 Jahre vor dieser Untersuchung bilateral adrenalektomiert worden. Die
Patientinnen mit dem M.Addison und der partiellen Nebennierenrindeninsuffizienz zeigten nach Corticotropin-Stimulation keinen bzw. nur einen geringen Anstieg der Cortisolkonzentration im Serum. Die Ürinproben dieser Patienten wurden im 3:Stunden-Abstand über 48 Stunden gesammelt.

Der mögliche Einfluß von Dexamethason auf den Tagesgang der Katecholaminausscheidung wurde an 8 gesunden Probanden untersucht, die um $10.00 \mathrm{Uhr}$ des ersten Versuchstages $6 \mathrm{mg}$ Dexamethason, dann $3 \mathrm{mg}$ in 6stundigem Abstand bis $4.00 \mathrm{Uhr}$ des übernächsten Tages einnahmeñ. Diese Probanden konnten sich im Klinikgelände frei bewegen. Die Urinproben wurden in 3-Stunden-Intervallen, beginnend um 9.00 Uhr über 24 Stunden, gesammelt. Den Zeitablauf des Versuchs gibt Abbildung 1 schematisch wieder.

Die Urinproben wurden nach der Probennahme angesäuert und bis zur Analyse bei $-25^{\circ} \mathrm{C}$ aufbewahrt.

\section{Bestimmungsverfahren}

Die Bestimmung der Katecholaminkonzentration erfolgte mit der Einisotopenderivatmethode nach Peuler \& Johnson (10), die wie folgt modifiziert wurde: $50 \mu$ U Urin, der je nach Katecholaminkonzentration 1:10 bis $1: 100$ mit bidest. Wasser verdünnt werden mußte, wurde pro Bestimmung eingesetzt. Zur Leerwertermittlung wurde bidest. Wasser verwendet. Die Trennung der tritiummarkierten Verbindungen erfolgte mittels Hochdruckflüssigkeitschromatographie anstelle der Dünnschichtchromatographie. Zur weiteren Abtrennung von unspezifischer Aktivität wurde die das 3-Methoxy tyramin enthaltende Fraktion acetyliert und das Reaktionsprodukt bei pH 5,4 mit Toluol ausgeschüttelt (11). Die Auswertung erfolgte über einen internen Katecholaminstandard. Die bei Verwèndung von Plasma teilweise auftretende Inhibierung der enzymatischen Reaktion konnte bei Verwendung von verdünntem Urin nicht beobach tet werden.

Bei jeder Bestimmungsserie wurden selbst hergestellte Kontrollurinproben mitanalysiert. Die Ergebnisse dieser statistischen Qualitätskontrolle sind in Tabelle 2 wiedergegeben.

Die an Kontrollurinproben ermittelten Schwankungen sind ein $\mathrm{Ma} ß$ für die methodisch bedingte Störung der wiedergegebenen

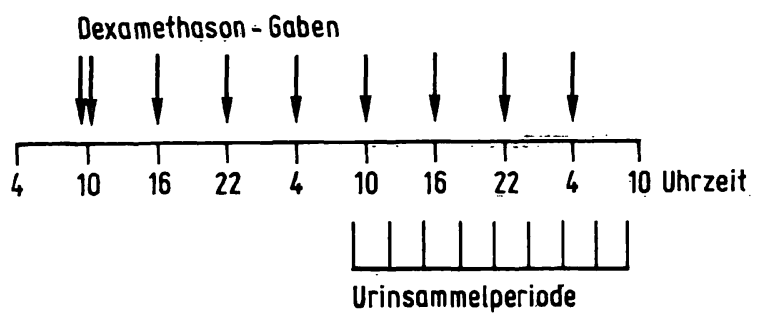

Abb. 1. Zeitablauf der Dexamethason-Gaben und Urinsammelperioden bei 8 gesunden Probanden.

Tab. 1. Klinische Daten der untersuchten Patienten.

Die Ergebnisse der Patienten H.W. und K.H. wurden einer Publikation von Knoll \& Wisser (9) entnommen. Bei den Ausscheidungen bedeuten die 1. und 2. Zahl Ausscheidungen am 1. und 2. Tag.

\begin{tabular}{|c|c|c|c|c|c|c|c|}
\hline \multirow[t]{3}{*}{ Pat. } & \multirow{3}{*}{$\begin{array}{l}\text { Alter } \\
\text { (Jah- } \\
\text { re) }\end{array}$} & \multirow[t]{3}{*}{ Diagnose } & \multirow{3}{*}{$\begin{array}{l}\text { Tumor- } \\
\text { gewicht }\end{array}$} & \multicolumn{4}{|c|}{ Ausscheidung } \\
\hline & & & & Noradrenalin & Adrenalin & Dopamin & $\begin{array}{l}\text { Vanillin- } \\
\text { mandel- } \\
\text { säure }\end{array}$ \\
\hline & & & & $(\mu \mathrm{g} / 24 \mathrm{~h})$ & $(\mu \mathrm{g} / 24 \mathrm{~h})$ & $(\mu \mathrm{g} / 24 \mathrm{~h})$ & $(\mathrm{mg} / 24 \mathrm{~h})$ \\
\hline $\begin{array}{l}\text { B.R. } \\
\text { G.M. } \\
\text { H.W. } \\
\text { K.H. } \\
\text { E.L. } \\
\text { Sch.R. } \\
\text { D.A. }\end{array}$ & $\begin{array}{l}36 \\
65 \\
55 \\
35 \\
64 \\
45 \\
21\end{array}$ & $\begin{array}{l}\text { Phäochromocytom } \\
\text { Phäochromocytom } \\
\text { Phäochromocytom } \\
\text { Rezidiv Neuroblastom } \\
\text { M. Addison } \\
\text { partielle Nebennierenrinden-Insuffizienz } \\
\text { bilaterale Adrenalektomie }\end{array}$ & $\begin{array}{r}100 \\
1130 \\
325 \\
-\end{array}$ & $\begin{array}{c}321 / 350 \\
1634 / 3437 \\
339 / 427 \\
2430 / 2630 \\
19,3 / 20,8 \\
22,4 / 20,8 \\
12,9 / 15,4\end{array}$ & $\begin{array}{c}422 / 452 \\
1436 / 1855 \\
363 / 438 \\
- \\
5,1 / 6,4 \\
5,5 / 3,9 \\
2,0 / 2,2\end{array}$ & $\begin{array}{c}338 / 358 \\
- \\
- \\
27400 / 27700 \\
218 / 248 \\
360 / 352 \\
488 / 493\end{array}$ & $\begin{array}{l}40 / 42 \\
153 / 295 \\
74 / 93 \\
92 / 68 \\
5,6 / 5,0 \\
5,7 / 5,0 \\
5,8 / 5,8\end{array}$ \\
\hline
\end{tabular}


Tab. 2. Ergebnis der Präzisionskontrolle mit selbst hergestellten Kontrollurinproben.

\begin{tabular}{llcc}
\hline \multirow{2}{*}{$\begin{array}{llc}\text { Bestand- } \\
\text { teil }\end{array}$} & $\begin{array}{l}\text { Statistische } \\
\text { Kenngröße }\end{array}$ & \multicolumn{2}{c}{ Streuung } \\
& & in der Serie & von Tag zu Tag \\
\hline Nor- & $\overline{\mathrm{x}}(\mu \mathrm{g} / 1)$ & 56,7 & 57,0 \\
adrenalin & $\mathrm{s}(\mu \mathrm{g} / \mathrm{l})$ & 2,6 & 4,0 \\
& $\mathrm{VK}(\%)$ & 4,5 & 6,9 \\
& $\mathrm{n}$ & 10 Einfachbest. & 10 Doppelbest. \\
Adrenalin & $\overline{\mathrm{x}}(\mu \mathrm{g} / 1)$ & 12,3 & 12,2 \\
& $\mathrm{~s}(\mu \mathrm{g} / 1)$ & 0,5 & 1,0 \\
& $\mathrm{VK}(\%)$ & 4,6 & 8,3 \\
& $\mathrm{n}$ & 10 Einfachbest. & 10 Doppelbest. \\
Dopamin & $\overline{\mathrm{x}}(\mu \mathrm{g} / 1)$ & 330 & \\
& $\mathrm{~s}(\mu \mathrm{g} / 1)$ & 10 & 331 \\
& $\mathrm{VK}(\%)$ & 3,3 & 17 \\
& $\mathrm{n}$ & 10 Einfachbest. & 10 Doppelbest. \\
& & & 5,1 \\
Vanillin- & $\overline{\mathrm{x}}(\mathrm{mg} / 1)$ & 3,9 & 3,9 \\
mandel- & $\mathrm{s}(\mathrm{mg} / 1)$ & 0,05 & 0,11 \\
säure & $\mathrm{VK}(\%)$ & 1,2 & 2,8 \\
& $\mathrm{n}$ & 19 Einfachbest. & 19 Doppelbest. \\
\hline
\end{tabular}

Versuchsergebnisse. Wie die Ergebnisse zeigen, liegt die methodische Streuung bei 5-8\%. Dies ist deutlich niedriger als die biologische Streuung. Ein mit 3-Stunden-Urinproben durchgeführter Vergleich zwischen radioenzymatischer und fluorometrischer Katecholaminbestimmung nach Weil-Malherbe (12) zeigte gute Übereinstimmung bezüglich der Noradrenalinkonzentration im Normalbereich ( $n=26, r=0,990)$. Radioenzymatisch wurden um 20-40\% höhere Adrenalinkonzentrationen als mit der fluorometrischen Methode $(n=26, r=0,934)$ bestimmt.

Die Vanillinmandelsäure wurde photometrisch - nach Extraktion und Natriumperiodatoxidation - bestimmt (13).

\section{Ergebnisse und Diskussion}

In Tabelle 3 ist die 24-Stunden-Ausscheidung der Katecholamine und der Vanillinmandelsäure bei vier Normälpersonen zusammengefaßt.
Tab. 3. 24-Stundenausscheidung der Katecholamine und Vanillinmandelsäure bei 4 gesunden Probanden.

\begin{tabular}{|c|c|c|c|c|c|c|c|c|}
\hline \multirow[t]{2}{*}{$\begin{array}{l}\text { Ver- } \\
\text { suchs- } \\
\text { gruppe }\end{array}$} & \multicolumn{2}{|c|}{$\begin{array}{l}\text { Nor- } \\
\text { adrenalin } \\
(\mu g / 24 \mathrm{~h})\end{array}$} & \multicolumn{2}{|c|}{$\begin{array}{l}\text { Adrenalin } \\
(\mu \mathrm{g} / 24 \mathrm{~h})\end{array}$} & \multicolumn{2}{|c|}{$\begin{array}{l}\text { Dopamin } \\
(\mu \mathrm{g} / 24 \mathrm{~h})\end{array}$} & \multicolumn{2}{|c|}{$\begin{array}{l}\text { Vanillin- } \\
\text { mandelsäure } \\
(\mathrm{mg} / 24 \mathrm{~h})\end{array}$} \\
\hline & 1. & -2. & $\begin{array}{l}1 . \\
\text { Tag }\end{array}$ & $\begin{array}{l}2 . \\
\text { Tag }\end{array}$ & 1. & $\begin{array}{l}2 . \\
\text { Tag }\end{array}$ & $\begin{array}{l}1 . \\
\text { Tag }\end{array}$ & $\begin{array}{l}2 . \\
\text { Tag }\end{array}$ \\
\hline \multicolumn{9}{|l|}{$\begin{array}{l}\text { Pro- } \\
\text { banden }\end{array}$} \\
\hline $\begin{array}{l}\text { B.H. } \\
\text { O.H. } \\
\text { H.J. } \\
\text { K.W. }\end{array}$ & $\begin{array}{l}29,6 \\
26,4 \\
37,2 \\
37,1\end{array}$ & $\begin{array}{l}31,3 \\
28,8 \\
38,8 \\
37,1\end{array}$ & $\begin{array}{r}7,1 \\
11,5 \\
13,8 \\
9,9\end{array}$ & $\begin{array}{r}7,8 \\
13,0 \\
13,2 \\
9,9\end{array}$ & $\begin{array}{l}287 \\
295 \\
310 \\
430\end{array}$ & $\begin{array}{l}323 \\
301 \\
305 \\
423\end{array}$ & $\begin{array}{l}6,1 \\
5,0 \\
6,7 \\
7,2\end{array}$ & $\begin{array}{l}4,9 \\
4,9 \\
5,5 \\
7,3\end{array}$ \\
\hline
\end{tabular}

Aus Tabelle 3 ist zu entnehmen, daß die interindividuellen Unterschiede innerhalb der Kontrollgruppe z.T. beträchtlich sind. Dies gilt nicht für den intraindividuellen Vergleich von Tag zu Tag. Um die interindividuellen Unterschiede auszugleichen, wurde der Gleichwert der Schwingung, ausgedrückt durch das arithmetische Mittel aller von 8.00 bis $8.00 \mathrm{Uhr}$ gemessenen Werte, für jede Versuchsperson und jeden Versuchstag bestimmt. Für jeden Einzelwert wurde anschließend die prozentuale Abweichung vom jeweiligen Gleichwert berechnet. Die Ergebnisse sind in Abbildung 2 zusammengefaßt, die den Tagesgang der Katecholamin- und Vanillinmandelsäureausscheidung bei der Kontrollgruppe zeigt.

Es fällt auf, daß die Maxima und die Minima asymmetrisch zueinander sind, d.h. nicht genau 12 Stunden voneinander entfernt liegen. Für das Noradrenalin und Adrenalin wird ein Maximum morgens zwischen 10.00 Uhr und 12.00 Uhr und ein weiteres Maximum, vor allem für das Adrenalin in der gleichen Größenordnung zwischen $16.00 \mathrm{Uhr}$ und $18.00 \mathrm{Uhr}$ bestimmt. Das Minimum liegt zwischen 22.00 Uhr und 4.00 Uhr nachts.

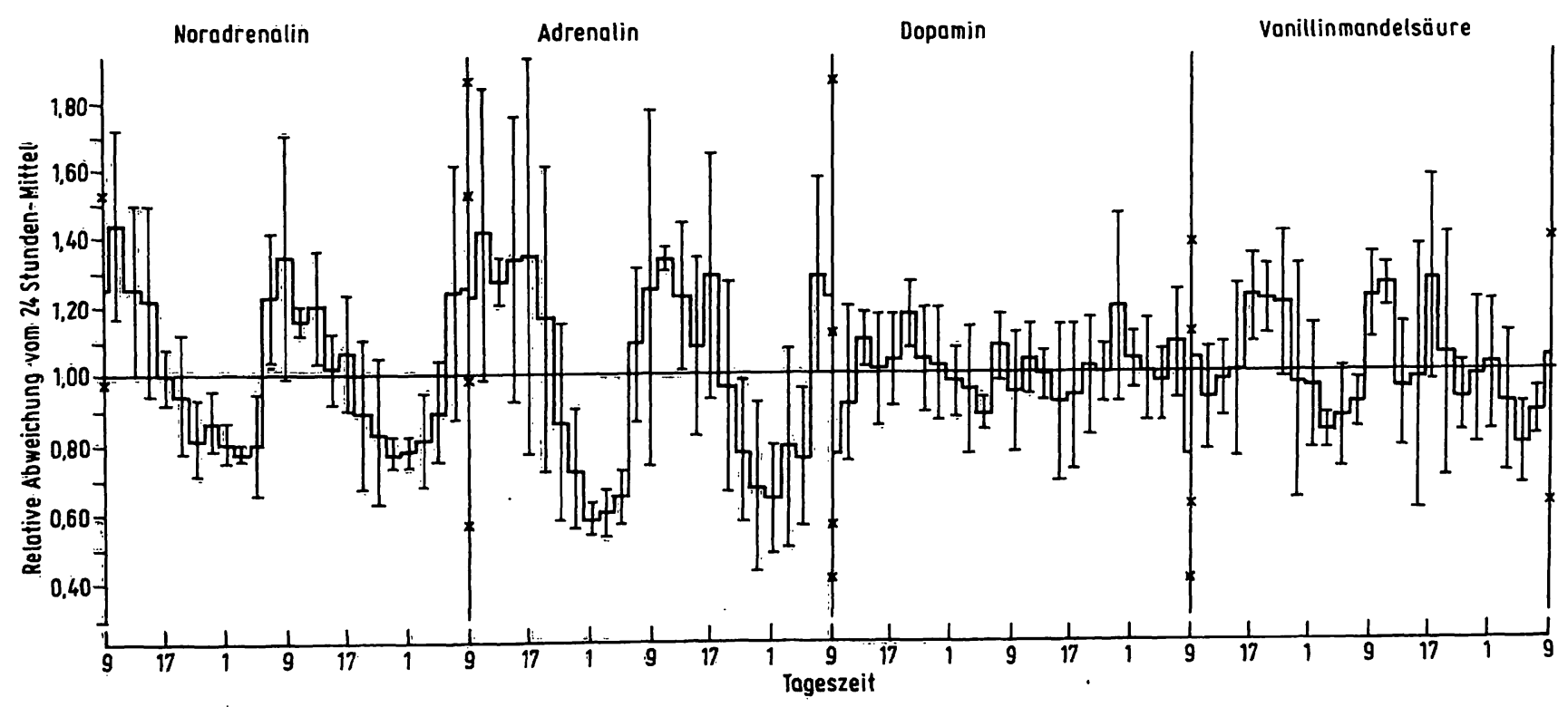

Abb. 2. Tageszeitabhängigkeit der Ausscheiđung der Katecholamine und Vanillinmandelsäure. Mittelwerte von 4 gesunden Probanden. Der 2s-Bereich wird durch die senkrechten Balken markiert. 
Dopamin zeigt einen gering ausgeprägten Rhythmus. Eine Phasenverschiebung zwischen Noradrenalin und Adrenalin ist nicht zu erkennen. Auffällig ist, daß die Schwingungsbreiten für das Noradrenalin und Adrenalin größer als für das Dopamin und die Vanillinmandelsäure sind. Die Schwingungsbreite wurde aus der Differenz zwischen Maximal- und Minimalwert eines Tages berechnet. Für Adrenalin und Noradrenalin wurden im Mittel Werte von $70 \%$, für Dopamin und Vanillinmandelsäure Werte von 30-40\% erhalten. Aber auch hier können erhebliche Unterschiede zwischen den einzelnen Probanden auftreten. Dieser Befund der gering ausgeprägten Rhythmik des Dopamins stimmt mit unseren früheren Ergebnissen überein, wobei die Vanillinmandelsäure ebenfalls bezüglich der Schwingungsbreiten ein dem Dopamin ähnliches Verhalten zeigte $(2,14)$.

Die Absolutwerte der Ausscheidungen der Katecholamine der untersuchten Patienten im 24-Stundenurin sind in Tabelle 1 wiedergegeben. Die Tageszeitabhängig- keit der Urinausscheidung von Adrenalin, Noradrenalin und Vanillinmandelsäure der vier Patienten mit erhöhter Katecholaminausscheidung zeigt Abbildung 3.

Vergleicht man diese Daten mit denen von Abbildung 2, so sieht man, daß die circadiane Rhythmik bei den $\mathrm{Pa}$ tienten mit erhöhter Katecholaminausscheidung nicht aufgehoben ist und daß die Schwingungsbreiten von Adrenalin und Noradrenalin derjenigen der Vanillinmandelsäure ähnlich sind. Dieser Befund stụitzt dịe Hypothese, daß die unterschiedlichen Schwingungsbreiten vop Adrenalin und Noradrenalin auf der einen Seite, sowie von Dopamin und Vanillinmandelsäure auf der anderēn Seite durch ihre Herkunft aus einer unterschiedlich großen Anzahl von Kompartimenten bedingt ist. Das Maximum der Ausscheidung der gemessenen Substanzen ist gegenüber gesunden Probanden zeitlich verspätet. Dies wird vor allem bei der Ausscheidung des Noradrenalins und Adrenalins deutlich.

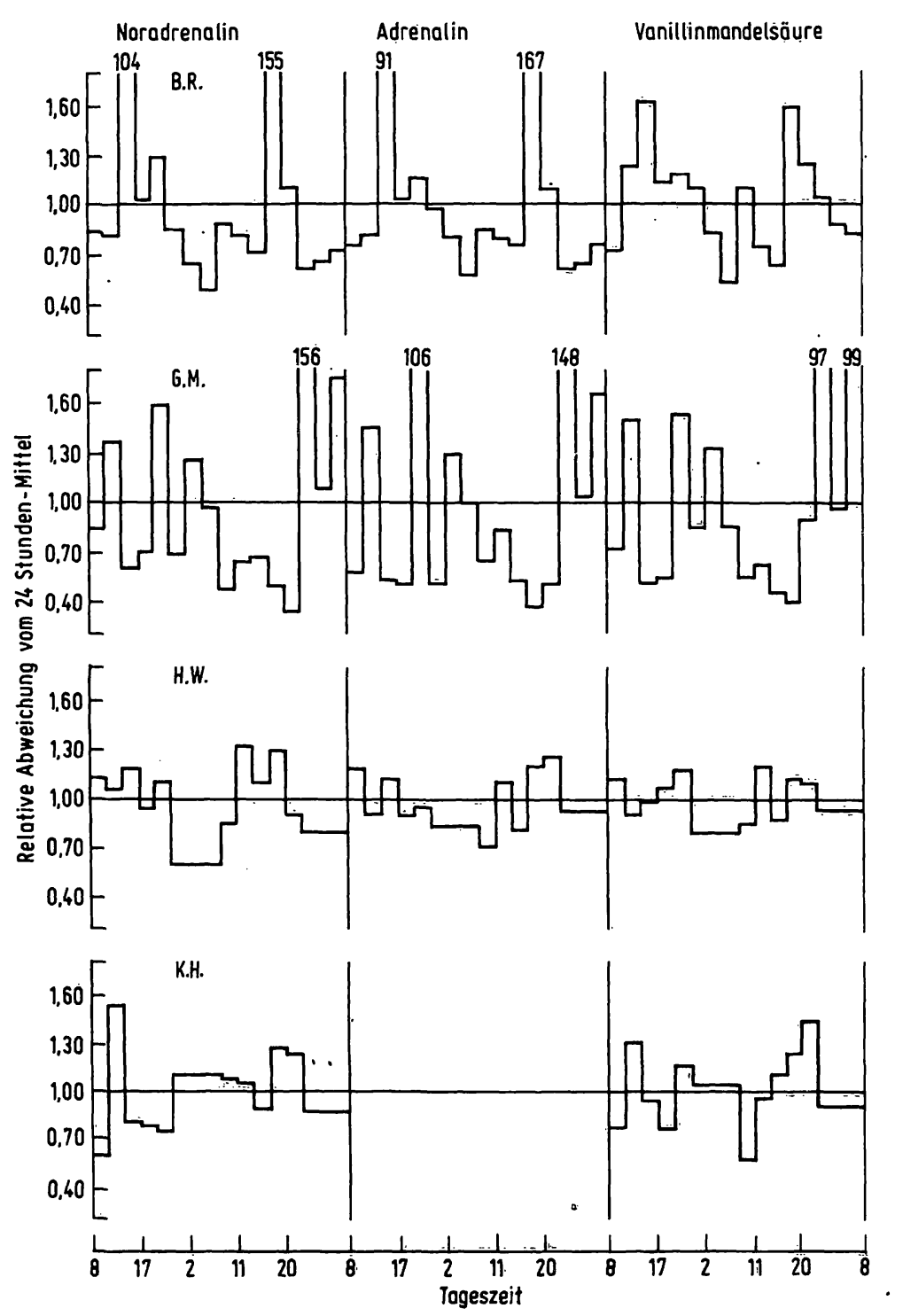

Abb. 3. Tageszeitabhängigkeit der Ausscheidung von Adrenalin, Noradrenalin und Vanillinmandelsäure bei 3 Patienten-mit operativ gesichertem Phäochromocytom und einem Patienten mit einem Rezidiv nach operativ entferntem Neuroblastom. 
In Abbildung 4 sind die Ergebnisse für die Patienten mit einer gestörten bzw. aufgehobenen Nebennierenrindenfunktion wiedergegeben.

Die Messungen über einen Zeitraum von 48 Stunden zeigen mit einer guten Reproduzierbarkeit die im Vergleich zu den gesunden Probanden unveränderte Tageszeitabhängigkeit der Katecholaminausscheidung. Insbesondere konnte bei der bilateral adrenalektomierten Patientin keine Änderung der Amplitude und der Phase beim Adrenalin im Vergleich mit den gesunden Probanden festgestellt werden. Dies ist nicht selbstverständlich, da das von dieser Patientin ausgeschiedene Adrenalin mit Sicherheit aus einem anderen Kompartiment stammt als das der gesunden Versuchspersonen. Der Absolutwert der Ausscheidung liegt aber für das Adrenalin und Noradrenalin an der unteren Normgrenze.

Wie einleitend erwähnt, konnten Wurtman \& Axelrod $(6,7)$ bei hypophysektomierten Ratten bei Gabe von Corticotropin bzw. Dexamethason über eine vermehrte Enzymneubildung eine Erhöhung der Phenylethanolamin-N-Methyltransferase-Aktivität der Nebenniere nachweisen. Die folgende Abbildung 5 gibt die Ergebnisse der Untersuchung an 8 gesunden Probanden über 24 Stunden wieder.

Während sich bei dieser Gabe von Dexamethason der normale nächtliche Anstieg der Serumtestosteronkonzentration unterdrücken ließ (8), zeigen die Ergebnisse
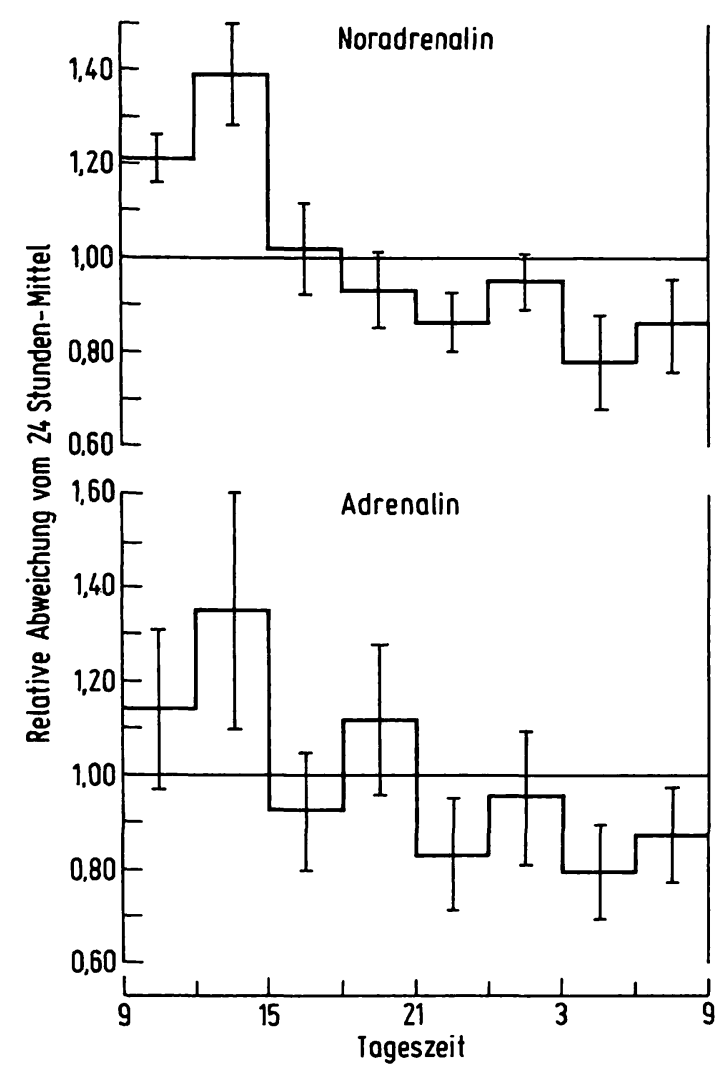

Abb. 5. Tageszeitabhängigkeit der Ausscheidung von Adrenalin und Noradrenalin unter Dexamethason-Gabe. (Mittelwerte von 8 gesunden Probanden).

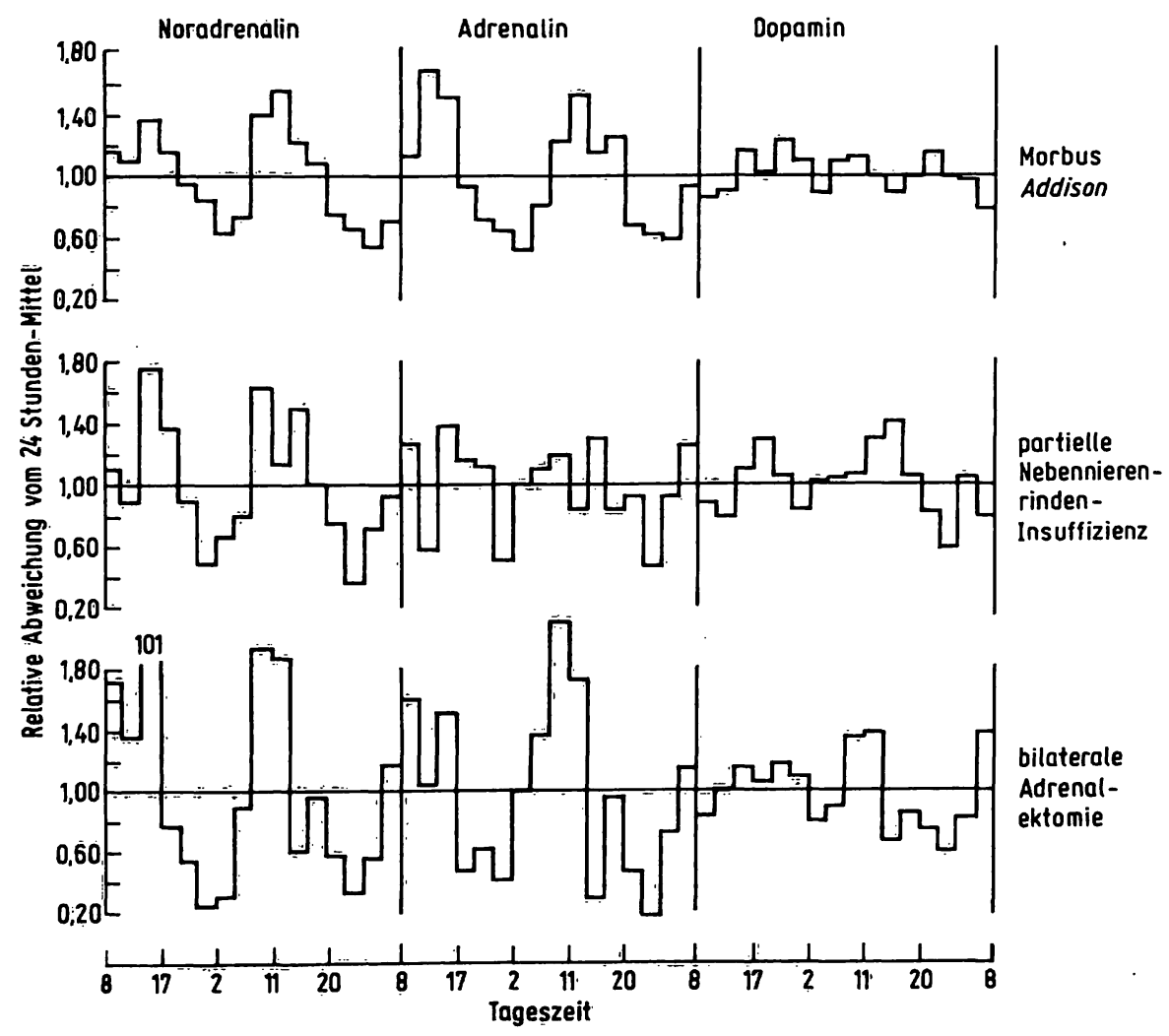

Abb. 4. Circadiane Rhythmik der Urinausscheidung von Adrenalin, Noradrenalin und Dopamin bei 3 Patienten mit gestörter Nebennierenrindenfunktion. 
für das Adrenalin und Noradrenalin keine Änderungen der circadianen Rhythmik. Wie die Ergebnisse der Patienten mit Nebenniereninsuffizienz zeigen, die unter einer Langzeitbehandlung mit Cortisolacetat standen, führt auch diese zu keiner Veränderung der circadianen Rhythmik.

Insgesamt läßt sich feststellen, daß sich sowohl bei einer gestörten Funktion des Nebennierenmarks, der Neben-

\section{Literatur}

1. Euler, U. S. v., Hellner-Björkman, S. \& Orwen, I. (1955), Acta Phy siol. Scand. 33, Suppl. 118, 10-16.

2. Aschoff, J., Fatranska, M., Giedke, H., Doerr, P., Stamm, D. \& Wisser, H. (1970), Science 171, 213-215.

3. Levi, L. (1966), Särtryck ur Försvarsmedicin 2, 3-8.

4. Wurtman, R. J. (1966), in: Catecholamines, Little Brown, Boston.

5. Euler, U. S. v., Franksson, C. \& Hellström, J. (1953), Acta Physiol. Scand. 31, 1-5.

6. Wurtman, R. J. \& Axelrod, J. (1965), Science 150, 14641466.

7. Wurtman, R. J. \& Axelrod, J. (1966), J. Biol. Chem. 241, 2301-2305. nierenrinde und bèi medikamentöser Beeinflussung der Nebenniere keine Änderung der circadianen Rhythmik der Katecholamine feststellen läßßst.

Ausnahmen bilden die Patienten mit Phäochromocytom, bei denen die Schwingungsbreiten von Adrenalin und Noradrenalin denen der Vanillinmandelsäure ähṇlicher werden, was mit eigenen früheren Untersuchungen übereinstimmt (9).

8. Doerr, P. \& Pirke, K. M. (1979), J. Steroid Biochem. 10, $81-86$.

9. Wisser, H. \& Knoll, E. (1975), Clin. Chim. Acta 59, 1-7.

10. Peuler, J. D. \& Johnson, G. A. (1977), Life Sciences 21, 625-636.

11. Bosak, J., Knoll, E., Ratge, D. \& Wisser, H. (1980), diese Z. $18,413-421$.

12. Weil-Malherbe, H. (1968), Methods Biochem. Anal. 16, $293-326$.

13. Wisser, H. \& Stamm, D. (1970), diese Z. 8, 21-26.

14. Wisser, H., Doerr, P., Stamm, D., Fatranska, M., Giedke, H. \& Wever, R. (1973), Klin. Wochenschr. 51, 242-246.

Prof. Dr. Dr. H. Wisser Abteilung für Klinische Chemie Robert-Bosch-Krankenhaus Auerbachstraße 110 D-7000 Stuttgart 50 